\title{
Spin conservation and Fermi liquid near a ferromagnetic quantum critical point
}

\author{
Andrey V. Chubukov ${ }^{1,3}$ and Dmitrii L. Maslov ${ }^{2,3}$ \\ 1 Department of Physics, University of Wisconsin-Madison, \\ 1150 University Ave., Madison, WI 53706-1390* \\ 2 Department of Physics, University of Florida, P. O. Box 118440, Gainesville, FL 32611-8440* \\ ${ }^{3}$ Max-Planck-Institut für Physik komplexer Systeme, D-01187 Dresden, Germany
}

(Dated: October 29, 2018)

\begin{abstract}
We propose a new low-energy theory for itinerant fermions near a ferromagnetic quantum critical point. We show that the full low-energy model includes, in addition to conventional interaction via spin fluctuations, another type of interaction, whose presence is crucial for the theory to satisfy $S U(2)$ spin conservation. We demonstrate the consistency between a loop-wise expansion and a Fermi liquid description for the full model. We further show that, prior to the ferromagnetic instability, the system develops a Pomeranchuk-type instability into a state with zero magnetization but with $p$-wave deformations of the Fermi surfaces of spin-up and -down electrons (a spin nematic).
\end{abstract}

PACS numbers: 71.10. Ay, 71.10 Pm

Introduction Conservation laws are powerful tools to study the behavior of interacting fermions. The Hamiltonian for a system of fermions with non-relativistic interactions is $S U(2)$ invariant and preserves the total charge and spin of particles. In this Letter, we analyze the consequences of spin conservation for effective low-energy theories, which describe the behavior of itinerant systems near a $q=0$ Pomeranchuk instability. In particular, we focus on the effective theory of a ferromagnetic quantum critical point (FM QCP). It has been long assumed that a FM QCP is well described by the spin-fermion model 1 (SFM) with interaction only in the spin channel, mediated by small $q$ collective spin excitations (for a review, see Refs. 2,3).

Here, we report two results. First, we show that the SFM is fundamentally incomplete as it violates spin conservation. We obtain a proper low-energy model which obeys spin conservation and show that in this new model the interaction is mediated by both spin and charge bosons; the charge component of the interaction is of the same strength as the spin component. We show that the perturbation theory for this model is consistent with the Fermi liquid (FL) description, which is based on conservation laws. This consistency does not hold in the SFM, as it has been recently emphasized in Ref. 4 .

Second, we show that, within the new model, the system develops an instability toward a $p$-wave spin nematic state,$\frac{5,6}{6}$ in which the net magnetization is absent but the Fermi surfaces of spin-up and spin-down electrons are shifted in opposite directions. For not too long-range interaction in the spin channel, this instability occurs not only before the Stoner instability into a ferromagnetic state but also before other known instabilities near a FM QCP - a first order transition, helical magnetic order, or p-wave superconductivity. $\underline{3,7,8,9,10}$

A $p$-wave spin nematic state, first introduced by Hirsch, $\underline{\underline{5}}$ has been studied in detail in recent years $\underline{\underline{6}}$ It was identified as the ground state of a particular lattice model,$\underline{5}$ but whether such a state can be realized in a rotationally and $S U(2)$-spin invariant model was not clear.
Our result shows that such a state emerges in an ordinary isotropic FL tuned to a FM QCP.

Spin conservation in the SFM. The SFM model was introduced as a minimal model near a magnetic QCP $\stackrel{1,11}{\underline{1}}$ It describes low-energy fermions with an effective spinspin interaction

$$
\Gamma_{\alpha \beta ; \gamma \delta}^{\Omega}\left(\mathbf{k}, \omega_{k} ; \mathbf{p}, \omega_{p}\right)=\frac{1}{2 \nu Z} \frac{1}{\delta+(a q)^{2}+\frac{|\Omega|}{v_{F} q}} \vec{\sigma}_{\alpha \delta} \cdot \vec{\sigma}_{\beta \gamma} .
$$

Here, $\mathbf{q}=\mathbf{k}-\mathbf{p}, \Omega=\omega_{k}-\omega_{p}$ are the relative momenta and Matsubara frequencies, respectively, $\delta$ measures the distance from the QCP, $\nu$ is the density of states, and $Z$ is the fermionic residue. The superscript $\Omega$ implies that Eq. (1) is obtained by integrating out fermions outside the Fermi surface (FS) but contains no contribution from fermions at the FS - the latter is obtained within the FL theory which takes $\Gamma^{\Omega}$ an input $\underline{12}$ The main structure of Eq. (11) is obtained already within the RPA ${ }^{2,13,14}$ (see Fig. 1a); an additional factor of $1 / Z$ arises from renormalization of $\Gamma^{\Omega}$ beyond the RPA. ${ }^{14}$

The effects of the interaction $\Gamma^{\Omega}$ can be analyzed in two ways - either within a loop-wise expansion in $\Gamma^{\Omega}$ or with the FL theory built upon conservation laws. In a model which satisfies conservation laws, the results of the two approaches must be identical. We first show that they are not in the SFM. For definiteness, we consider below the case of $D=2$, but our results are also valid for $D=$ 3 . Loop-wise expansions of bosonic and fermionic selfenergies have been performed by a number of authors, and the results relevant to our discussion are ${ }^{15,16}$

$$
\frac{m^{*}}{m} \approx \frac{1}{Z}=\lambda \equiv \frac{3}{4 a k_{F} \sqrt{\delta}} ; \chi_{s}=\chi_{s}^{0} \propto \frac{1}{\delta} .
$$

Observe that the static uniform spin susceptibility is not renormalized by the interaction at low energies.

To obtain the results for $m^{*} / m$ and $\chi_{s}$ in the FL theory, one has to convert $\Gamma^{\Omega}$ into the Landau function $g_{\alpha \beta ; \gamma \delta}(\mathbf{k}, \mathbf{p})=Z^{2}\left(m^{*} / m\right) \Gamma_{\alpha \beta ; \gamma \delta}^{\Omega}(\mathbf{k}, 0 ; \mathbf{p}, 0)$, obtain charge and spin harmonics of $g_{\alpha \beta ; \gamma \delta}=g_{c} \delta_{\alpha \gamma} \delta_{\beta \delta}+g_{s} \vec{\sigma}_{\alpha \gamma} \cdot \vec{\sigma}_{\beta \delta}$ for 
FIG. 1: a) Ladder diagrams for $\Gamma^{\Omega}$. Summing up these diagrams for a contact interaction $U$ (dashed line) and rearranging spin indices, one obtains the effective spin-spin interaction near the critical point (wavy line), where $U m / 2 \pi \approx$ 1. The rest of the diagrams (not shown) eliminate the instability in the charge channel. b) Renormalization of the spinfermion vertex. The last two diagrams are the AslamazovLarkin-type (AL) contributions which restore spin conservation. c) The full effective interaction $\Gamma^{\Omega \text {,full }}$ with contributions from the direct spin-spin interaction and AL-type processes.
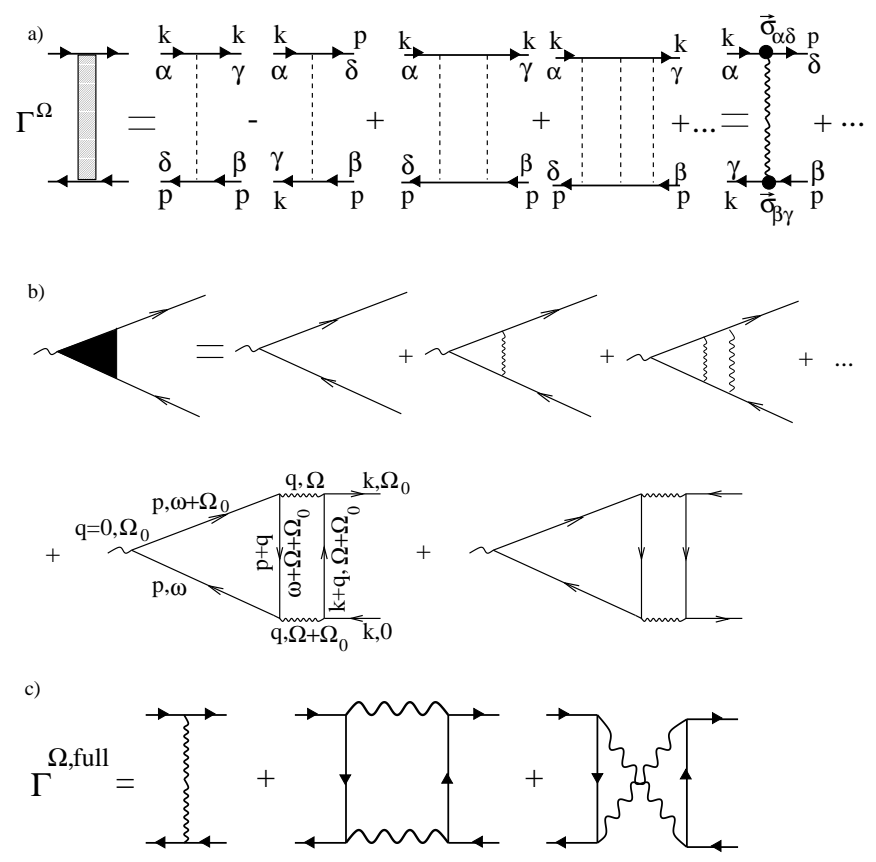

the particles on the FS $\left(|\mathbf{k}|=|\mathbf{p}|=k_{F}, \omega_{k}=\omega_{p}=0\right)$, and use familiar FL relations $m^{*} / m=1+g_{c, 1}$ and $\chi_{s}=\chi_{s}^{0}\left(1+g_{c, 1}\right) /\left(1+g_{s, 0}\right)$. Carrying out this procedure, we find $g_{c, 1}=\lambda$ and $g_{s, 0}=-\lambda / 3$, i.e.,

$$
\frac{m^{*}}{m}=\lambda ; \chi_{s}=\chi_{s}^{0} \frac{1+\lambda}{1-\frac{\lambda}{3}} .
$$

The result for $m^{*} / m$ is consistent with Eq. (2) but the result for $\chi_{s}$ is not. Moreover, Eq. (3) shows that $\chi_{s}$ has a spurious divergence at $\lambda=3$, i.e., at finite distance from the QCP.

This disagreement between the two approaches is related to a violation of spin conservation in the SFM. To see this, we compute the spin and charge susceptibilities in the SFM at a small but finite frequency $\Omega$ and zero momentum $q$. The Ward identities require that $\chi_{c, s}(q=0, \Omega)=0$. We show that $\chi_{s}(q=0, \Omega) \neq 0$, although $\chi_{c}(q=0, \Omega)=0$.

The effect of the interaction with collective modes on the spin and charge susceptibilities can be parameterized as

$$
\chi_{c, s}(q=0, \Omega)=\chi_{c, s}^{0}\left(1-\frac{\Lambda_{c, s}}{1+\lambda}\right),
$$

where $\Lambda_{c, s}$ are the fully renormalized fermion-boson vertices normalized to $\Lambda_{c, s}=1$ for non-interacting fermions. Self-consistency requires that $\Lambda_{c, s}$ are obtained within the same RPA approximation as Eq. (1) itself. The RPA series [cf. Fig. 1b)] is geometric; the $n$-th order term is given by $(\lambda /(1+\lambda))^{n}$ for the charge vertex and $(-1 / 3)^{n}(\lambda /(1+\lambda))^{n}$ for the spin vertex 17 The difference comes from spin summation: the charge vertex contains a combination $\delta_{\alpha \beta} \vec{\sigma}_{\alpha \gamma} \cdot \vec{\sigma}_{\delta \beta}=3 \delta_{\gamma \delta}$, while the spin vertex contains $\sigma_{\alpha \beta}^{i} \vec{\sigma}_{\alpha \gamma} \cdot \vec{\sigma}_{\delta \beta}=-\sigma_{\gamma \delta}^{i}$. Summing up the series, we find that $\Lambda_{c}=1+\lambda$ and $\Lambda_{s}=(1+\lambda) /(1+4 \lambda / 3)$. Substituting $\Lambda_{c, s}$ into Eq. (4), we find that charge susceptibility $\chi_{s}(q=0, \omega)$ vanishes, as it should, but $\chi_{s}(q=0, \omega)$ does not, which implies that spin conservation is violated.

This violation has a physical explanation $\underline{17}$ In the SFM, electron spins are split into spins of itinerant electrons, $\mathbf{s}=c_{\alpha}^{\dagger} \vec{\sigma}_{\alpha \beta} c_{\beta}$ and spins $\mathbf{S}$ of collective bosons. In the $S U(2)$ symmetric case, the fermion-boson coupling $\mathbf{S} \cdot \mathbf{S}$ flips $s^{z}$. As a result, $\mathbf{s}$ is not conserved separately from $\mathbf{S}$.

Because of spin non-conservation, the SFM was argued 17 to be applicable only in the regime when conservation laws do not matter, i.e., when relevant momenta are much larger than relevant frequencies. Some physical properties described by the SFM, e.g., mass renormalizaton, superconducting and other instabilities of a paramagnetic state, come from fermions from that range and are therefore adequately described within the SFM $\cong .16$ Still, the violation of spin conservation implies that the SFM model is fundamentally incomplete as the $S U(2)$ invariance is not broken in a paramagnetic phase at any energy. A correct low-energy model, which includes all relevant interactions, should obey spin conservation.

Spin-conserving low-energy model. We now construct such a model by going beyond the ladder approximation for the vertex corrections $\Lambda_{c}$ and $\Lambda_{s}$. It turns out that the important diagrams are the Aslamazov-Larkin (AL) type-diagrams encountered, e.g., in the theory of superconducting fluctuations $\underline{18}$ To second order, there are two such diagrams shown in the last line of Fig. 1 $b)$. For the charge vertex, the two diagrams cancel each other $\stackrel{19}{=}$ For the spin vertex, they add up and contribute

$$
\begin{aligned}
I_{\mathrm{AL}}= & \frac{1}{2 \nu Z^{2}} \int \frac{d q q}{2 \pi} \int \frac{d \Omega}{2 \pi} \int \frac{d \theta_{\mathbf{p q}}}{2 \pi} \int \frac{\theta_{\mathbf{k q}}}{2 \pi} \int \frac{d \omega}{2 \pi} \int d \epsilon_{p} G(\mathbf{p}, \omega) G\left(\mathbf{p}, \omega+\Omega_{0}\right) G\left(\mathbf{k}_{F}+\mathbf{q}, \Omega+\Omega_{0}\right) \\
& \times\left[G\left(\mathbf{p}+\mathbf{q}, \omega+\Omega+\Omega_{0}\right)+G(\mathbf{p}-\mathbf{q}, \omega-\Omega)\right] \bar{\Gamma}(\mathbf{q}, \Omega) \bar{\Gamma}\left(\mathbf{q}, \Omega+\Omega_{0}\right)
\end{aligned}
$$


Here, $G(\mathbf{k}, \omega)=Z /\left[i \omega-\epsilon_{k}\left(m / m^{*}\right)\right]$ is the Green's function of FL quasiparticles, $\epsilon_{k}=v_{F}\left(k-k_{F}\right), m^{*} / m=$ $Z^{-1}=1+\lambda, \quad \theta_{\mathbf{l m}}=\angle(\mathbf{l}, \mathbf{m})$, and $\bar{\Gamma}(\mathbf{q}, \Omega)=$ $\left(\delta+(a q)^{2}+|\Omega| / v_{F} q\right)^{-1}$. Although $I_{\mathrm{AL}}$ is formally a two-loop correction in $\bar{\Gamma}$, its contribution is actually of the same order as from the one-loop correction. To see this, we observe that relevant $\epsilon_{p}$ in Eq. (5) are small (of order $\Omega_{0}$ ) while $q$ is finite. Hence, the integration over $\epsilon_{p}$ involves effectively only the first two Green's functions in the first line. For the poles of these two Green's functions to be in different half-planes, the range of $\omega$ must be bounded by the external frequency $-\Omega_{0}<\omega<0$. Hence, one can neglect $\omega$ and $\Omega_{0}$ in the remainder of the integral. The angular integrations now factorize and each of them gives a factor of $\left(m^{*} / m\right) / v_{F} q$. The remaining integrals over $q$ and $\Omega$ have a scaling property $\int d q \int d\left(\Omega / v_{F} q\right) \bar{\Gamma}^{2}\left(q, \Omega / v_{F} q\right)=\int d q \bar{\Gamma}(q, 0) \sim \lambda$. A more accurate calculation yields $I_{\mathrm{AL}}=(4 / 3) \lambda /(1+\lambda)$ (the denominator comes from power-counting factors of $Z$ ). Combining $I_{\mathrm{AL}}$ with the one-loop ladder vertex correction, which contributes $-(1 / 3) \lambda /(1+\lambda)$, we find that the effective one-loop correction to $\Lambda_{s}$ becomes $\lambda /(1+\lambda)$, which is exactly the same result as the correction to $\Lambda_{c}$. It can be readily shown that the effective $n$th other contribution to $\Lambda_{s}$ now is $(\lambda /(1+\lambda))^{n}$, again the same as for $\Lambda_{c}$.
Summing up the series, we obtain that now $\Lambda_{s}=1+\lambda$, which means that $\chi_{s}\left(0, \Omega_{0} \rightarrow 0\right)=0$ and thus spin conservation is restored. Other two-loop vertex corrections are small as $\max \left\{\Omega_{\mathrm{FL}} / E_{F}, \Omega_{0} / \Omega_{\mathrm{FL}}\right\}$, where $\Omega_{\mathrm{FL}} \propto \delta^{3 / 2}$ is the upper boundary for the FL description.

For completeness, we also analyzed spin conservation right at a FM QCP, where the fermionic self-energy $\Sigma(\omega) \propto \omega^{2 / 3}$. The calculations at QCP are more involved, and to verify the spin Ward identity one has to solve an integral equation for the full vertex. For the spin vertex $\Gamma_{s}$ we find the same result as above: the AL-type diagrams restore spin conservation.

Once we have established that AL blocks play the role of an effective single bosonic propagator, it is obvious that these blocks also give additional contributions to the FL vertex $\Gamma_{\alpha \beta ; \gamma \delta}^{\Omega}(\mathbf{k}, 0 ; \mathbf{p}, 0)$ and, hence, to Landau parameters. These contributions, shown Fig. 1 $c$ ), add an extra term of the structure $\left(3 \delta_{\alpha \delta} \delta_{\beta \gamma}-\vec{\sigma}_{\alpha \delta} \cdot \vec{\sigma}_{\beta \gamma}\right) F(\mathbf{k}+\mathbf{p})$ to $\Gamma_{\alpha \beta ; \gamma \delta}^{\Omega}(\mathbf{k}, 0 ; \mathbf{p}, 0)$. Although $F(\mathbf{k}+\mathbf{p})$ is a rather complex function of its argument, one can show that its partial harmonics are the same as those of the RPA-like kernel $1 /\left(\delta+a^{2}(\mathbf{k}+\mathbf{p})^{2}\right)$. This statement is true for harmonics with $n \ll \lambda$ which are the only ones we need. Combining Eq. (1) with the AL contribution, we obtain for the full vertex near a FM QCP

$$
\Gamma_{\alpha \beta ; \gamma \delta}^{\Omega, \text { full }}(\mathbf{k}, 0 ; \mathbf{p}, 0)=\frac{1}{2 \nu Z}\left(\frac{\vec{\sigma}_{\alpha \delta} \cdot \vec{\sigma}_{\beta \gamma}}{\delta+a^{2}(\mathbf{k}-\mathbf{p})^{2}}+\frac{3 \delta_{\alpha \delta} \delta_{\beta \gamma}-\vec{\sigma}_{\alpha \delta} \cdot \vec{\sigma}_{\beta \gamma}}{\delta+a^{2}(\mathbf{k}+\mathbf{p})^{2}}\right) .
$$

Two comments are now in order. First, the AL term in Eq. (6) has both spin and charge components and the latter is by no means negligible. In other words, a spinconserving low-energy model contains singular interactions in both spin and charge channels. Second, the AL term has the same structure as the direct spin-fluctuation exchange term, but becomes singular at small total momentum $(\mathbf{k} \approx-\mathbf{p})$ rather than at small transferred momentum $(\mathbf{k} \approx \mathbf{p})$.

Fermi liquid near a FM QCP. We now construct the new Landau function $g_{\alpha \beta ; \gamma \delta}^{\text {full }}(\mathbf{k}, \mathbf{p})=$ $Z^{2}\left(m^{*} / m\right) \Gamma_{\alpha \beta ; \gamma \delta}^{\omega, \text { full }}(\mathbf{k}, 0 ; \mathbf{p}, 0)=g_{c}^{\text {full }} \delta_{\alpha \gamma} \delta_{\beta \delta}+g_{s}^{\text {full }} \vec{\sigma}_{\alpha \gamma} \cdot \vec{\sigma}_{\beta \delta}$ from the effective interaction $\Gamma^{\Omega \text {,full }}$. After some standard spin algebra, we obtain

$$
\begin{aligned}
& g_{\alpha \beta ; \gamma \delta}^{\text {full }}(\theta)=\frac{3}{2} \frac{\delta_{\alpha \gamma} \delta_{\beta \delta}}{\delta+4\left(a k_{F}\right)^{2} \sin ^{2} \theta / 2}+\frac{1}{2} \vec{\sigma}_{\alpha \gamma} \cdot \vec{\sigma}_{\beta \delta} \times \\
& \left(\frac{4}{\delta+4\left(a k_{F}\right)^{2} \cos ^{2} \theta / 2}-\frac{1}{\delta+4\left(a k_{F}\right)^{2} \sin ^{2} \theta / 2}\right),
\end{aligned}
$$

where $\theta=\angle(\mathbf{k}, \mathbf{p})$. Evaluating $g_{c, 1}^{\text {full }}$ and $g_{s, 0}^{\text {full }}$, we obtain $g_{c, 1}^{\text {full }}=g_{s, 0}^{\text {full }} \approx \lambda$ for $\lambda \gg 1$, hence

$$
m^{*} / m=\lambda ; \chi_{s}=\chi_{s}^{0} \frac{1+\lambda}{1+\lambda}=\chi_{s}^{0} .
$$

These two results are now in full agreement with those of the loop-wise expansion, Eq. (2).

The FL theory with the Landau function from Eq. (7) is rather non-trivial. First, not only $g_{c, 1}$ but all charge components with $n \ll \lambda$ diverge near criticality in the same way: $g_{c, n}^{\text {full }}=(3 / 2 \pi) \int_{0}^{\pi} d \theta \cos (n \theta) /(\delta+$ $\left.4 a^{2} k_{F}^{2} \sin ^{2} \theta / 2\right) \approx \lambda$ (see Fig. 2). The divergence of an infinite set of Landau parameters also exists near a charge QCP $\stackrel{14}{\underline{14}}$ This does not lead to dramatic consequences as the divergence of $g_{c, n>1}$ is compensated by that of $m^{*} / m=1+g_{c, 1}$, and the charge susceptibilities $\chi_{c, n}=\chi_{c, n}^{0}\left(m^{*} / m\right) /\left(1+g_{c, n}^{\text {full }}\right)=\chi_{c, n}^{0}(1+\lambda) /(1+\lambda)=\chi_{c, n}^{0}$ remain intact.

Second, and more important, we see from Eq. (7) that all odd spin Landau parameters are negative and diverge at the FM QCP. Since a Pomeranchuk instability occurs when the corresponding Landau parameter approaches -1, it implies that a spin Pomeranchuk instability with finite angular momentum occurs before the isotropic, Stoner-like instability. Indeed, to leading order 
FIG. 2: (Color on-line.) Schematic evolution of the partial components of the full Landau function [Eq. (77)] vs the inverse mass renormalization parameter $\lambda^{-1}$. All charge and even spin Landau components are positive but diverge near a FM QCP, while odd spin components are negative. The spin nematic component $g_{s, 1}$ reaches the Pomeranchuk critical value of -1 first.

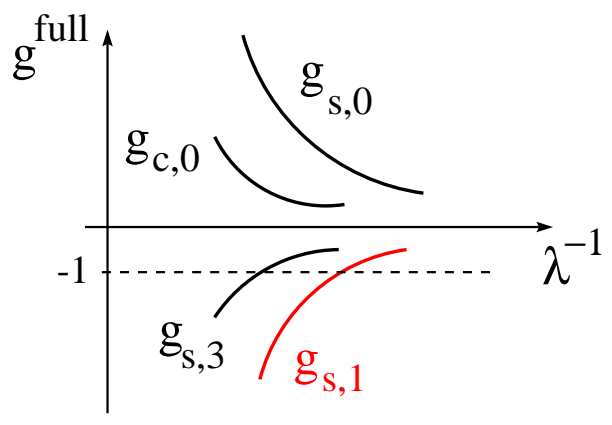

in $1 / \lambda$, we have from Eq. (77) $g_{s, n}^{\text {full }}=\lambda \delta_{n, 2 m}-\frac{5}{3} \lambda \delta_{n, 2 m+1}$. For $n=2 m+1, g_{s, n}^{\text {full }}=-5 \lambda / 3$, and $g_{s, n}^{\text {full }}$ approaches -1 at $\lambda=3 / 5$. To discriminate between states with different angular momenta, one needs to evaluate the Landau parameters beyond the leading order in $1 / \lambda$. Doing so, we find, quite naturally, that $g_{s, 1}^{\text {full }}$ approaches -1 first. Therefore, the leading instability corresponds to a $p$-wave spin nematic state. Such a state in itinerant Fermi systems has recently attracted significant attention. $\underline{\underline{6}}$ Two different phases $(\alpha$ and $\beta)$-the analogs of $A$ and $B$ phases of $3 \mathrm{He}-$ have been identified, but only the $\alpha$ phase is actually stable. This phase is characterized by a $p$-wave spin order parameter $\Delta_{s}=\sum_{\mathbf{k}} f(k)\left(c_{\mathbf{k} \uparrow}^{\dagger} c_{\mathbf{k} \uparrow}-c_{\mathbf{k} \downarrow}^{\dagger} c_{\mathbf{k} \downarrow}\right) \cos \theta_{\mathbf{k}}$, where $f(k)$ is sharply peaked at the FS. This state has zero magnetization but the centers of FSs of spin-up and spin-down electrons are separated by finite momentum.

As we have already mentioned, a $p$-wave instability competes with more "conventional" instabilities of a FL near the FM QCP - p-wave superconductivity, a first order transition and a transition into a helical magnetic phase. Which one occurs first depends on the parameters of the model. Controllable calculations are possible only when the range of interaction in the spin channel is large, i.e., for $a k_{F} \gg 1$. According to Ref. 9, the most relevant out of "conventional" instabilities is the first order transition, which occurs at $\delta=\delta_{c r}^{1} \approx 0.21 /\left(a k_{F}\right)$. The $p$-wave Pomeranchuk instability occurs at $\delta=\delta_{c r}^{p}=25 / 16\left(a k_{F}\right)^{2}$. Although $\delta_{c r}^{p}$ is smaller than $\delta_{c r}^{1}$ by power-counting, the two critical values coincide at large enough value of $a k_{F} \approx 7.44$, which implies that for a realistic case of $a k_{F} \sim 1$, the $p$-wave instability comes first.

Finally, we notice that $S U(2)$ spin invariance is only approximate in real systems and broken by, e.g., spinorbit (SO) coupling. This breaks the exact relation between the ladder and AL-type vertex corrections, but the AL term in $\Gamma^{\Omega}$ still remains of the same order as the bare spin interaction, and, as long as SO coupling is small, the system still undergoes a $p$-wave spin-nematic instability at $\lambda \sim 1$.

To conclude, we considered the behavior of an $S U(2)$ invariant itinerant fermionic system near a ferromagnetic quantum critical point. We showed that the spin-fermion model, traditionally used to describe this behavior, does not satisfy $S U(2)$ spin conservation. We obtained a spinand charge-conserving low-energy model for a FM QCP. This model has singular interactions in both spin and charge channels, as opposed to the SFM which considers interaction only in the spin channel. We constructed a FL theory for such a model and found that the system undergoes a Pomeranchuk instability into a $p$-wave spin nematic state before a FM QCP is reached.

We acknowledge helpful discussions with C. Castellani, A. Green, I. Eremin, E. Fradkin, P. Hirsch, H.-Y. Kee, Eun-Ah Kim, Y.-B. Kim, S. Kivelson, M. Lawler, A. Rosch, A. Varlamov, T. Vojta, and C. Wu, support from NSF-DMR-0604406 (A. V. Ch.) and NSF-DMR-0908029 (D. L. M.).
* Permanent address.

1 J. A. Hertz, Phys. Rev. B 14, 1165 (1976); A. J. Millis, Phys. Rev. B 48, 7183 (1993); T. Moriya, Spin Fluctuations in Itinerant Electron Magnetism (Springer, Berlin, 1985).

2 D. J. Scalapino, Phys. Rep. 250, 329 (1995)

3 H. v. Löhneysen, A. Rosch, M. Vojta, and P. Wölfle, Rev. Mod. Phys. 79, 1015 (2007) and references therein.

${ }^{4}$ V. A. Khodel, J. W. Clark, and M. V. Zverev, arXiv:0904.1509

5 J. Hirsch, Phys. Rev. B 41, 6820 (1990).

${ }^{6}$ C. Wu, K. Sun, E. Fradkin, S-C. Zhang, Phys. Rev. B 75, 115103 (2007) and references therein

7 K. B. Blagoev, J. R. Engelbrecht, and K. Bedell, Phys. Rev. Lett. 82, 133 (1999); A. Chubukov et al, Phys. Rev.
Lett. 90, 077002 (2003); P. Monthoux and G. G. Lonzarich, Phys. Rev. B 69, 064517 (2004); M. Dzero and L. P. Gor'kov, Phys. Rev. B 69, 092501 (2004); A. A. Katanin, Phys. Rev. B 72, 035111 (2005).

8 D. Belitz, T. R. Kirkpatrick, and T. Vojta, Rev. Mod. Phys. 77579 (2005).

9 D. L. Maslov and A. V. Chubukov, Phys. Rev. B 79, 075112 (2009) and references therein.

10 G. J. Conduit, A. G. Green, and B. D. Simons, Phys. Rev. Lett. 103, 207201 (2009)

11 Ar. Abanov, A. V. Chubukov, and J. Schmalian, Adv. Phys. 52, 119 (2003).

12 A. A. Abrikosov, L. P. Gorkov, and I. E. Dzyaloshinski, Methods of quantum field theory in statistical physics, (Dover Publications, New York, 1963). 
13 N. F. Berk and J. R. Schrieffer, Phys. Rev. Lett. 17, 433 (1966).

14 D.L. Maslov and A.V. Chubukov, arXiv:0911.1251

15 P. A. Lee and N. Nagaosa, Phys. Rev. B 46, 5621 (1992); B. L. Altshuler, L. B. Ioffe, and A. J. Millis, Phys. Rev. B 50, 14048 (1994); C. Castellani, C. DiCastro, and M. Grilli, Phys. Rev. Lett. 75, 4650 (1995).

16 W. Metzner, D. Rohe, and S. Andergassen, Phys. Rev. Lett. 91, 066402 (2003); A. A. Katanin, A. P. Kampf, and V. Yu. Irkhin, Phys. Rev. B 71, 085105 (2005); A.
V. Chubukov, Phys. Rev. B 71, 224431 (2005); J. Rech, C. Pépin, and A. V. Chubukov, Phys. Rev. B 74, 195126 (2006).

17 A. V. Chubukov, Phys. Rev. B 72, 085113 (2005).

18 L. G. Aslamazov and A. I. Larkin, Phys. Lett. A 26, 238, (1968); Sov. Phys.: Solid State 10, 875 (1968).

19 The cancellation for the charge case holds only for $v_{F} q / \Omega \rightarrow 0$; see Sung Sik Lee, Phys. Rev. B 80, 165102 (2009), 